\title{
UM NOVO ENFOQUE PARA A TRADUÇÃO NO ENSINO COMUNICATIVO DE LÍNGUAS ${ }^{1}$
}

\author{
Carmen CUÉLLAR LÁZARO, Universidade de Valladolid \\ Tradução de Nylcéa Thereza DE SIQUEIRA PEDRA - UFPR
}

\begin{abstract}
RESUMO: No presente artigo pretende-se reivindicar o lugar da tradução no ensino de línguas estrangeiras. Para tanto, depois de uma breve apresentação de como a tradução foi tratada nos diferentes métodos de ensino e de como o afastamento da língua materna da sala de línguas resultou no abandono de atividades tradutórias em sala de aula, apresenta-se como a tradução é assumida pelo método comunicativo. Depois dessas considerações, expõe-se o resultado da pesquisa realizada em diferentes manuais de ensino de espanhol como língua estrangeira para analisar como a língua materna e a tradução aparecem contempladas neles.
\end{abstract}

PALAVRAS-CHAVE: tradução; método comunicativo; ensino de espanhol como língua estrangeira.

ABSTRACT: In this article we intend to claim the place of translation in the teaching of foreign languages. Thus, after a brief presentation of how the translation tool was treated in the different teaching methods and how the removal of the mother tongue from the language classroom resulted in the abandonment of translation activities in the classroom, translation is presented as it is assumed by the communicative approach. After these considerations, the results of the research carried out in different teaching manuals of Spanish as a foreign language are presented in order to analyze how the mother tongue and the translation appear contemplated in them.

KEYWORDS: translation; communicative approach; teaching Spanish as a foreign language.

\section{INTRODUÇÃO}

O papel atribuído à tradução foi variando ao longo da história do ensino de línguas estrangeiras. Os métodos tradicionais de ensino, os chamados métodos passivos, utilizavam como modelo a metodologia empregada para o ensino de línguas clássicas e atribuíam um papel de destaque à tradução. O objetivo não era se comunicar na língua aprendida, mas alcançar o significado do texto, geralmente literário, mediante a análise dos diferentes aspectos gramaticais exercitados com a tradução. Posteriormente, com os métodos diretos, a língua materna desapareceu do processo de ensino-aprendizagem de língua estrangeira e se excluiu qualquer atividade relacionada com a tradução. Hoje em dia, alguns professores têm tentado reintegrar a tradução ao ensino de línguas estrangeiras no marco do ensino comunicativo.

\footnotetext{
${ }^{1}$ Artigo publicado originalmente em língua espanhola na Revista Hermēneus. Revista de Traducción e Interpretación, n.6, 2004. ISSN: 1139-7489. Disponível em: $<<$ https://dialnet.unirioja.es/servlet/articulo?codigo=1027635>>
} 
O principal objetivo deste artigo é, precisamente, expor o novo papel outorgado à tradução na aprendizagem de línguas sob a ótica do método comunicativo. Para isso, será feita uma breve reflexão sobre a evolução metodológica do uso da tradução dentro das salas de aula de línguas estrangeiras nas últimas décadas. Também serão apresentadas as opiniões de diferentes teóricos partidários da reintegração da tradução ao processo de ensino-aprendizagem de uma língua estrangeira. Finalmente, observaremos, a partir de exemplos, em que consiste esse novo enfoque da tradução em sala de aula.

\section{2. "LEIA E TRADUZA" VERSUS "NÃO PENSE NA SUA LÍNGUA, NÃO TRADUZA"}

Há duas máximas que resumem a evolução que os métodos empregados nas aulas de línguas estrangeiras sofreram nas últimas décadas: "leia e traduza" e "não pense não sua língua, não traduza". Ambas constituem os polos opostos dessa evolução. Por um lado, aulas baseadas em ler e traduzir o lido, textos basicamente literários, descontextualizados da vida real, com listas de vocabulário e estruturas gramaticais. Ninguém usava a língua estrangeira na sala, a não ser os personagens dos diálogos apresentados em fitas K7 e, esporadicamente, o professor.

Por outro lado, estavam as aulas que, desde o primeiro dia, aconteciam na língua estrangeira, pretendendo uma aproximação "natural" e "direta" à nova língua. Os métodos "diretos" desterraram a tradução das aulas de língua, fazendo a língua materna também desaparecer do processo de aquisição de uma língua estrangeira. No entanto, faz tempo que esses métodos deixaram de ser a panaceia das salas de aula. A aquisição de uma língua estrangeira é muito diferente da aprendizagem de uma língua estrangeira e, ainda que o professor peça para seus alunos não traduzirem, estes o fazem automaticamente e de maneira interiorizada, principalmente nos níveis iniciais. Como já comentou um estudioso do tema "a sombra da língua materna é grande" (SÜSS, 1997, p.59).

$\mathrm{Na}$ prática de ensino atual, coexistem diferentes correntes metodológicas de orientação comunicativa. O objetivo é que o aluno seja capaz de compreender e produzir atos de fala adequados a uma intenção comunicativa e a uma situação concreta (HURTADO ALBIR, 1996, p.11). Para alcançar esse objetivo, dedica-se uma atenção especial ao desenvolvimento de tarefas e atividades que criem, na sala de aula, situações comunicativas, alicerces do processo de aprendizagem. No método comunicativo, é o 
processo, e não o resultado, que ganha protagonismo. Assim, são analisadas as características individuais (fatores cognitivos e afetivos) que certamente repercutem na aprendizagem do aluno. Pensa-se, junto com o aluno, em quais estratégias utiliza para aprender, fomentando a autoaprendizagem e dando-lhe consciência de que ele é o responsável pela sua aprendizagem, de que aprender uma língua também é "aprender a aprender" essa língua. O protagonismo que assumem os estudantes no processo de ensino-aprendizagem de uma língua estrangeira traz consigo a possibilidade de que reflitam sobre as diferenças e semelhanças entre a sua realidade e a do outro. Aprender uma nova língua supõe entrar em contato com uma realidade social e cultural nova, por isso, a interculturalidade é outro fator que merece destaque na orientação comunicativa da aprendizagem de línguas.

Nesse contexto, dentro do âmbito do ensino comunicativo de línguas, alguns pesquisadores defendem a reconsideração do papel da tradução na aprendizagem de uma língua estrangeira.

\title{
3. A TRADUÇÃO E O ENSINO DE LÍNGUAS ESTRANGEIRAS: "RECONCILIAÇÃO'"
}

A professora francesa Elisabeth Lavault foi, em meados dos anos oitenta do século XX, uma das primeiras a reivindicar a reconsideração da tradução na sala de aula:

\begin{abstract}
Abandonada pelas metodologias, ignorada pelas instituições oficiais de ensino, a tradução está onipresente na aprendizagem das línguas estrangeiras no ensino fundamental e médio, mesmo que seja apenas como tradução silenciosa. Já não é hora de considerar de forma positiva a reintegração da tradução no ensino de línguas? Parece-nos importante olhar em duas direções: para a tradução profissional e escolas de tradução, onde a prática intensiva de uma tradução pedagógica resultou em uma teorização sobre o ato de traduzir, que só pode enriquecer a nossa abordagem; e para as novas abordagens de ensino de línguas que têm superado um número significativo de clichês, reconsiderando o papel da língua materna na aprendizagem de línguas estrangeiras (LAVAULT, 1985, p.48)
\end{abstract}

No âmbito da língua inglesa, destaca-se o trabalho de Duff (1989) que também apresenta argumentos para o uso da tradução em sala de aula:

Todos nós temos uma língua materna ou primeira língua. Isso molda nosso modo de pensar e, até certo ponto, nosso uso da língua estrangeira (pronúncia, escolha de palavras, tom, ordem das palavras, etc.). A tradução ajuda-nos a entender melhor a influência da língua e a corrigir os erros de hábitos que passam despercebidos [...] Fora da sala de aula - em escritórios, bancos, fábricas, lojas e aeroportos - a tradução acontece o tempo todo. Por 
que não dentro da sala de aula? [...] A competência linguística é um sistema bidirecional, não unidirecional. Precisamos ser capazes de nos comunicar das duas maneiras: dentro e fora da língua estrangeira. [...] O material da tradução é o autêntico, não é linguagem "inventada". [...] é uma atividade que, por sua própria natureza, convida à especulação e à discussão [...] desenvolvendo três habilidades essenciais para toda a aprendizagem de línguas: precisão, clareza e flexibilidade. (DUFF, 1989, p.6)

$\mathrm{Na}$ Espanha, a professora Amparo Hurtado Albir também defende a "reconciliação" entre a tradução e ensino de línguas estrangeiras e a sua reintegração ao contexto do ensino comunicativo. Justifica a sua defesa com quatro argumentos: 1) o novo lugar da tradução na sociedade atual; 2) o novo enfoque dado às reflexões em torno da tradução com o desenvolvimento dos estudos da tradução; 3) a nova concepção de ensino da tradução e 4) a reconsideração do papel da língua materna no processo de aprendizagem da língua estrangeira (HURTADO ALBIR, 1994, p.69)².

É certo que o século XX foi considerado como a "era da tradução" (CUÉLLARLÁZARO, 2000, p.15). A atividade tradutória alcança nesse século um avanço espetacular, motivado pela intensificação das relações internacionais e pelos avanços tecnológicos. No auge da tradução, principalmente na segunda metade do século XX, também aumentam os estudos teóricos relacionados a ela. Mas será na década de oitenta que os estudos sobre a tradução se consolidam como uma disciplina com identidade própria. Nascem os estudos da tradução com uma característica essencial: a multidisciplinaridade ${ }^{3}$. Sua relação com outras disciplinas como a crítica literária, a linguística, a pedagogia, a sociologia, etc., permitiu uma aproximação a tradução desde diferentes perspectivas. Isso supôs não apenas uma diversidade terminológica (coexistem denominações como: linguística aplicada à tradução, ciência da tradução, etc.), mas também uma diversidade de enfoques metodológicos: os centrados na comparação das línguas, os enfoques textuais, os que se focam no processo de tradução, os que se fundamentam na tradução com um ato de comunicação, etc. ${ }^{4}$ Todos esses enfoques contribuem com aspectos que ajudam a compreender melhor a complexidade do processo tradutório. O propósito dessa reflexão é destacar a importância da

\footnotetext{
${ }^{2}$ Na mesma linha se encontram os estudos de Montaner Gutiérrez (1996) e De Arriba (2001). Outros trabalhos que se dedicam à reflexão sobre a tradução e o ensino de línguas: García Yebra (1985), Del Castillo (1986), Saéz Hermosilla (1986), Ballester Casado e Chamorro Guerrero (1993), Süss (1997), Rodríguez Castellano (1997) e Hernández (1998).

${ }^{3}$ A entrada da tradução como disciplina própria nas instituições universitárias acarretou uma série de problemas epistemológicos. Sobre a falta de consenso para definir os estudos da tradução no mundo científico conferir o estudo de Cuéllar-Lázaro (2000)

${ }^{4}$ São muitos os estudos dedicados à evolução da reflexão sobre a tradução (HURTADO ALBIR, 2001, p.99).
} 
"comunicação" na tradução, uma vez que é possível dizer que, hoje em dia, já há um consenso em assumir a função comunicativa da tradução: "as principais características da tradução são três: ser um ato comunicativo, uma operação entre textos (e não entre línguas) e um processo mental" (HURTADO ALBIR, 1994, p.72). A tradução é um ato de comunicação, se traduz para comunicar alguma coisa, "para que um destinatário que não entende a língua, e às vezes também a cultura, na qual está formulado o texto, oral ou escrito, tenha acesso a ele" (HURTADO ALBIR, 1994, p.72). Essa dimensão pragmática da tradução é a base do novo emprego da tradução nas aulas de língua sob uma orientação comunicativa. Trataremos disso na próxima seção.

\section{PARA AONDE VAMOS? UM NOVO ENFOQUE DA TRADUÇÃO}

A nova relação entre a tradução e o ensino de línguas estrangeiras traz consigo a premissa de que quando se recorra à tradução em sala de aula - seja como mecanismo de acesso ao significado da língua estrangeira (de maneira interiorizada ou explícita), seja como instrumento didático (tradução "pedagógica" ou tradução de textos) - isso não consista apenas em traduzir para entender, para alcançar o significado. Deve-se ter presente que, uma vez compreendido, o exercício de contraste pode nos ajudar a encontrar as interferências entre as línguas e evitá-las (HURTADO ALBIR, 1994, p.74). Além disso, o aluno deve se acostumar a traduzir no contexto, e não palavra por palavra, contrastando os meios que emprega cada língua para expressar uma mesma situação comunicativa. Essa é uma das contribuições da nova visão da tradução ao ensino comunicativo de línguas.

Mas até que ponto essas reflexões teóricas se refletem na sala de aula? Com o objetivo de responder a essa pergunta, fizemos a análise de um elemento de uso diário em sala de aula e que costuma ser espelho das concepções teóricas de sua época: os manuais didáticos. Foram analisados onze manuais de ensino de língua estrangeira, especificamente de espanhol como língua estrangeira, publicados entre 1995 e $2002^{5}$. O objetivo foi comprovar se esses manuais apresentavam atividades relacionadas à tradução e, se o faziam, que tipo e com qual objetivo. Do resultado do estudo se alcançam duas conclusões bastante significativas: por um lado, apesar da maioria dos manuais não fazerem menção à língua materna ou o fazerem de maneira esporádica, em três dos manuais analisados começa a se fazer eco, com maior ou menor clareza, da

\footnotetext{
${ }^{5}$ Os manuais analisados aparecem relacionados nas referências bibliográficas ao final do artigo.
} 
reintegração do papel da língua materna na aprendizagem de uma língua estrangeira, atribuindo-lhe uma função consciente na sala de aula ${ }^{6}$. E, por outro lado, o papel dado à tradução está claramente relacionado aos aspectos mais relevantes do método comunicativo.

Assim, encontram-se atividades que procuram motivar o aluno mediante $o$ interesse pela sua própria realidade propondo-lhe, por exemplo, traduzir as palavras ou expressões que utilize muito em sua língua para a língua estrangeira: "Pense em três palavras ou expressões que você use muito na sua língua e não conheça em espanhol. Pesquise como são ditas e escreva aqui." (Nuevo E/LE Inicial 1, Cuaderno de ejercicios, 2001, p.25), ou traduzir a sua citação favorita: "Pense na sua citação favorita ou na sua frase famosa favorita e traduza para o espanhol" (Nuevo E/LE Intermedio, Libro del alumno, 2002, p.87). Também se faz referência à cidade e ao país do aluno: "Você já sabe como se diz o nome do seu país e o gentilício dos seus habitantes em espanhol? Se não, pergunte para o seu professor. Pergunte também se existe uma tradução para o nome da sua cidade." (Gente 1, Libro del alumno, 1997, p.24). Também foram encontradas algumas atividades relacionadas a aspectos concretos da língua materna, como sua história, sua formação, seus dialetos, etc.: " $E$ a sua língua? Como foi criada? Que outras línguas participaram de sua formação? Você conhece alguma outra palavra que tenha origem em outra língua? Qual? (Gente 3, Libro del alumno, 2001, p.19). “E no seu país? Quantas línguas são faladas? Existem dialetos? (Gente 2, Libro de trabajo y resumen gramatical, 2002, p.25). "Se fala da mesma maneira no norte e no sul do seu país? Quais as diferenças: pronúncia, léxico...? Se fala do mesmo jeito nos diferentes bairros da sua cidade?" (Rápido, Rápido, Libro del alumno, 2002, p.69). Esse tipo de atividade fomenta - principalmente em grupos plurilíngues - um elemento muito importante no contexto atual: o trabalho da interculturalidade em sala de aula (CUÉLLAR-LÁZARO, 2002).

Do mesmo modo, foram encontradas atividades que convidam o aluno a fazer uma reflexão sobre o seu processo de aprendizagem, sobre a maneira de aprender uma língua estrangeira, a meta-aprendizagem. A tradução aparece nesse contexto como uma possível estratégia de aprendizagem. São realizadas atividades que convidam o aluno a

\footnotetext{
${ }^{6}$ Os três manuais são: Gente. Curso comunicativo basado en el enfoque por tareas; Nuevo E/LE. Curso de español para extranjeros e Rápido, Rápido. Curso intensivo de español. A continuação, serão apresentados alguns exemplos encontrados nesses três manuais e também nos outros manuais analisados. Por questão de direitos autorais, não colocamos os recortes das atividades, mas em todas elas aparece a fonte de referência para consulta.
} 
refletir sobre que estratégias utilizar para se lembrar de novos vocábulos, como, por exemplo, a tradução no seu contexto, deduzir o significado das novas palavras pela sua semelhança com as palavras de outras línguas, etc.: "O que você faz para se lembrar das palavras novas? Anota no caderno? Revê a lista algumas vezes? Tenta utilizá-las em contextos diferentes? Tenta se lembrar da sua pronúncia? Se você anota no seu caderno, coloca a tradução ao lado? Organiza as palavras de alguma maneira?” (Es español 3, Libro del alumno, 2001, p. 59). Também encontramos conselhos como: "Uma boa maneira de ampliar o seu vocabulário é criar o seu próprio dicionário, com tradução, desenho, palavras associativas ou qualquer outra técnica que seja útil para se lembrar das novas palavras." (Redes Nivel 1, Cuaderno de ejercicios, 2002, p. 55). Em outro manual, é proposta a seguinte atividade: “a) Marque quais dessas palavras você não entende e procure o significado. Aplique as estratégias que você considere mais apropriadas para memorizá-las. b) Copie no seu caderno as palavras que você não conhecia. Feche o livro e escreva a tradução delas na sua língua.” (Nuevo E/LE Intermedio, Libro del alumno, 2002, p. 55).

Ao mesmo tempo em que são fomentadas as estratégias de aprendizagem, também o são as de interação e comunicação, objetivando que o aluno alcance não apenas a competência linguística, mas também uma competência discursiva, estratégica e sociolinguística, isto é, uma competência comunicativa. Nesse sentido, a reconsideração do papel da língua materna no processo de aprendizagem de uma língua estrangeira traz consigo outro aspecto interessante: aproveitar no processo de aprendizagem de uma língua estrangeira as estratégias comunicativas, de compreensão e expressão que os alunos já desenvolveram em suas línguas maternas.

Começamos com as atividades que se centram fundamentalmente no desenvolvimento da competência linguística do aluno, em praticar a fonética, o vocabulário e a gramática. Geralmente não se limitam à mera tradução, mas convidam o aluno a refletir sobre as diferenças da sua língua materna. Isso é muito interessante porque será justamente nas diferenças onde o aluno encontrará as suas maiores dificuldades, interferências que dificultarão a sua aprendizagem. Só conseguirão superálas se tiverem conhecimento delas. Um exemplo disso poderia ser a formação dos números, aprendida nos níveis iniciais. Uma atividade propõe que o estudante pratique a compreensão e a expressão oral em espanhol do 100 ao 1.000.000: “a) Escute e identifique os números. b) Escute e repita. c) Diga os números em voz alta”, para, 
finalmente, convidar a uma reflexão interlíngua: “d) Em que se diferem da sua língua?” (Nuevo E/LE Inicial 1, Libro del alumno, 2001, p. 53).

Outras tarefas se centram em evitar as interferências derivadas da fonética como, por exemplo: "Qual é o som da letra B na sua língua? E da letra V? Escute estas frases e observe se em espanhol elas têm o mesmo som. Não se preocupe se não entender o significado delas. Depois de escutar, responda: as letras B e V são representadas pelo mesmo som? (Es español 2, Cuaderno de recursos y ejercicios, 2001, p. 42). Em outra atividade, na frente das letras que compõem o alfabeto da língua espanhola, pergunta-se ao aluno: "Quais letras não existem na sua língua? Diga para o professor." (Nuevo E/LE Inicial, Libro del alumno, 2001, p. 9). Considero que, no caso de alunos cuja língua materna não seja conhecida pelo professor, esse tipo de comentário pode ser de grande ajuda, uma vez que somente se o professor estiver consciente das dificuldades enfrentadas pelos alunos poderá pensar em atividades para praticar aqueles aspectos que sejam mais difíceis para eles.

Outra das novidades desse novo enfoque da tradução - como já comentamos - é a introdução de atividades que pretendem desenvolver no aluno uma competência discursiva, estratégica e sociolinguística que facilite sua competência comunicativa. Assim, são encontradas atividades para refletir sobre os diferentes registros da língua (familiar, coloquial, culta) comparando o espanhol com a língua materna dos estudantes: "Observe todos os textos que Mariano recebeu: estão escritos com o mesmo estilo, no mesmo tom? Em que se diferem um do outro? Por que você acha que isso acontece? Acontece o mesmo na sua língua?” (Gente 2, Libro del alumno, 1998, p.100); também são encontradas situações de contraste entre o uso de "tú" e "usted" em espanhol e na língua materna do aluno: "A escolha entre o uso de tú ou usted é muito difícil. Depende de muitos fatores. Observe as situações nos quadrinhos anteriores. Por que os personagens escolhem aquela forma de tratamento? Quais fatores interferem na decisão? Aconteceria o mesmo na sua língua? (Gente 1, Libro del alumno, 1997, p. 105); ou situações mais concretas como, por exemplo, a análise dos modos que cada língua emprega nos seguintes casos: "Relacione as reações com as situações que puderam provocá-las. Escreva o que você diria em sua língua em uma situação similar. As situações são: “a) ¡Oiga, camarero! ¿Quiere hacer el favor de atenderme? b) ¿Por qué no vamos a un restaurante italiano donde cantan tangos? c) ¿Qué guapa estás! d) ¡Qué bien te sienta el color rojo! e) Vamos a brindar por...f) ;Le he pedido merluza y 
esto no es merluza!", e as reações: "1. Perdone señor. Me he confundido. 2. ¡Chin chin! A la salud de... 3. ¿De verdad? 4. Un momentito,

por favor. 5. Eres un encanto. 6. ¡Mentiroso me queda horroroso! 7. ¡Qué horror! 8. ¡Basta!"7 (Primer Plano 3, Libro del alumno, 2002, p. 71). Resumidamente, trata-se de desenvolver no aluno estratégias de comunicação. Inclusive, algumas atividades são apresentadas com este título: "ESTRATÉGIAS DE COMUNICAÇÃO”, como é o caso da seguinte tarefa: "Em certas ocasiões precisamos de tempo para pensar no que vamos dizer. Por isso, usamos algumas expressões que nos permitem ter um tempo para preparar o que vamos falar. Outras vezes, repetimos a última coisa que falamos ou a pergunta que nos fizeram. Algumas das expressões utilizadas em espanhol são: “CCómo te diría yo?”, “La verdad es que...”, “Bueno, pues...” , e continua com a seguinte reflexão: "Você se lembra o que diz na sua língua nessas situações casos? Verifique com o professor se existem equivalentes em espanhol" (Nuevo E/LE Intermedio, Libro del alumno, 2002, p. 126).

Para concluir, observamos que o receio de que os alunos confrontem a língua que estão aprendendo com a sua língua materna está diminuindo. A língua materna do aluno adulto que inicia a aprendizagem de uma língua estrangeira é um elemento de interferência no processo de aprendizagem, mas também é uma bagagem linguística e cognitiva que pode facilitar a aprendizagem da língua (HURTADO ALBIR, 1988, p.74). Nesse contexto, a tradução se apresenta como um meio para o conhecimento das interferências e, a partir disso, como um instrumento para solucioná-las ${ }^{8}$.

\section{REFERÊNCIAS}

DE ARRIBA, Carlos. Traducción pedagógica. Uso de la traducción en la clase de lenguas extranjeras (alemán en secundaria), Tese de doutorado. Universidade de Barcelona, 2001.

\footnotetext{
${ }^{7}$ a) Ô, garçom! Quer fazer o favor de me atender? b) Por que não vamos a um restaurante italiano onde cantam tango? c) Que bonita você está! d) Você fica bem de vermelho! e) vamos fazer um brinde para... f) pedi pescada e isso não é pescada! E as reações: 1. Me desculpe, senhor, me confundi. 2. Tim-tim! Saúde! 3. Sério? 4. Um minutinho, por favor. 5. Que gentil! 6. Mentiroso! Fica horrível! 7. Que horror! 8. Chega!

${ }^{8}$ Durante muito tempo o erro, assim como a interferência, foi estudado como obstáculo, como algo negativo a ser evitado em sala de aula. O produto, o resultado, sempre foi o foco da aprendizagem, de ai o olhar negativo para o erro. No entanto, atualmente, no ensino comunicativo, reflete-se sobre a aprendizagem, a formação como processo e, nesse percurso, o erro é um companheiro inevitável, uma vez que não há aprendizado sem erro. Nesse sentido, tem-se uma visão positiva do erro, da interferência, somente conhecendo-os podemos evitá-los e, com isso, continuar avançando no processo de aprendizagem (DE LA TORRE, 1993, p.45).
} 
DE LA TORRE, Saturnino. Aprender de los errores. Editorial Escuela Española: Madrid, 1993.

DEL CASTILlO, María José. La traducción como soporte pedagógico. Actas del IV Congreso Nacional de Lingüística Aplicada. Universidad de Córdoba, 1986, p.168-183.

DUFF, Alan. Translation. Oxford University Press. Oxford, 1989.

GARCÍA YEBRA, Valentín. Traducción y enseñanza de las lenguas extranjeras. Actas del III Congreso Nacional de Lingüística Aplicada. Universidad de Valencia, 1989, p.143-155.

GRELLET, Françoise. Apprendre à traduire. Presses Universitaires de Nancy. Nancy, 1990.

HERNÁNDEZ, María Rosario. La traducción pedagógica en la clase de E/LE. Lengua y cultura en la enseñanza del español a extranjeros, Actas del VII Congreso de ASELE. Edição da Universidade de Castilla la Mancha: Cuenca, 1998, p.249-255.

HURTADO ALBIR, Amparo. La traducción en la enseñanza comunicativa. Cable no 1 , 1989, p.42-45.

Hacia un enfoque comunicativo de la traducción. II Jornadas Internacionales de Didáctica del español como lengua extranjera. Ministério da

Cultura: Madrid, 1988, p.53-79.

. Un nuevo enfoque de la traducción en la didáctica de las lenguas. Traducción, Interpretación, Lenguaje. Actas III Congreso Internacional Expolingua: Madrid, 1994, p.67-89.

La didáctica de la traducción. Evolución y estado actual. Perspectivas de la traducción inglés/español, Instituto de Ciencias de la Educación, Universidad de Valladolid: Valladolid, 1995.

Didáctica de segundas lenguas en los estudios de traducción. La enseñanza de la traducción. Universitat Jaume I: Castelló, 1996.

Madrid, 2001.

Traducción y Traductología. Introducción a la Traductología. Cátedra:

LAVAULT, Elisabeth. Fonction de la traduction en didactique des langues, Collection Traductologie, no.2. Didier Érudition: Paris, 1985.

MONTANER GUTIÉRREZ, Pedro. Tareas de traducción. La rehabilitación de la traducción en la didáctica del E/LE. Dissertação de Mestrado. Universidade de Barcelona, 1996.

RODRÍGUEZ CASTELLANO, José. La traducción como instrumento didáctico en la enseñanza del alemán. Actas de las I Jornadas de Jóvenes Traductores. Universidad de las Palmas de Gran Canaria. Palmas, 1997, p. 107-113. 
SÁEZ HERMOSILLA, Teresa. El concepto de traducción y sus aplicaciones pedagógicas. Cuadernos de Grado. Cáceres, año IV, nº 4, 1986.

SÜSS, Karl. La traducción en la enseñanza de idiomas. La palabra vertida. Investigaciones en torno a la traducción. Actas de los VI Encuentros en torno a la traducción. Universidad Complutense: Madrid, 1997, p.57-67.

FONTES

BOROBIO, Virgilio. Nuevo E/LE. Curso de español para extranjeros. Inicial 1. Libro del alumno. Ediciones SM: Madrid, 2001.

. Libro de ejercicios. Ediciones SM: Madrid, 2001.

Nuevo E/LE. Curso de español para extranjeros. Inicial 2. Libro del alumno. Ediciones SM: Madrid, 2002.

Libro de ejercicios. Ediciones SM: Madrid, 2002.

. Nuevo E/LE. Curso de español para extranjeros. Intermedio. Libro del alumno. Ediciones SM: Madrid, 2002.

Libro de ejercicios. Ediciones SM: Madrid, 2002.

CERROLAZA, Matilde (org.). Planet@ E/LE 1. Libro del alumno. Edelsa: Madrid, 1998.

. Libro de referencia gramatical: fichas y ejercicios. 1998.

.Planet@E/LE 2. Libro del alumno. Edelsa: Madrid, 1999.

. Libro de referencia gramatical: fichas y ejercicios. 1999.

Planet@E/LE 3. Libro del alumno. Edelsa: Madrid, 2000.

. Libro de referencia gramatical: fichas y ejercicios. 2000.

Planet@E/LE 4. Libro del alumno. Edelsa: Madrid, 2000.

Libro de referencia gramatical: fichas y ejercicios. 2000.

CHAMORRO, María Dolores (org.) Abanico. Curso avanzado de Español Lengua Extranjera. Libro del alumno. Difusión: Barcelona, 1998.

Cuaderno de ejercicios. Difusión: Barcelona, 1999.

INSTITUTO CERVANTES. Es español 1. Nivel inicial. Libro del alumno. Espasa: Madrid, 2001. 
REVISTA X, Curitiba, volume 14, n.2, p.145-157, 2019.

Cuaderno de recursos y ejercicios. Espasa: Madrid 2001.

INSTITUTO CERVANTES. Es español 2. Nivel intermedio. Libro del alumno. Espasa: Madrid, 2001.

. Cuaderno de recursos y ejercicios. Espasa: Madrid 2001.

INSTITUTO CERVANTES. Es español 3. Nivel avanzado. Libro del alumno. Espasa: Madrid, 2001.

MARCOS DE LA LOSA, María del Carmen. Punto final. Curso superior E.L.E. Edelsa: Madrid, 1997.

Cuaderno de recursos y ejercicios. Espasa: Madrid 2001.

OLIVA, Carmen. Redes. Curso de español para extranjeros. Nivel 1. Libro del alumno. Ediciones SM: Madrid, 2002.

Cuaderno de ejercicios. Ediciones SM: Madrid, 2002.

PALOMINO, María Ángeles. Primer Plano 1. Libro del alumno. Edelsa: Madrid, 2000.

. Cuaderno de ejercicios. Edelsa: Madrid, 2000.

. Primer Plano 2. Libro del alumno. Edelsa: Madrid, 2001.

. Cuaderno de ejercicios. Edelsa: Madrid, 2001.

. Primer Plano 3. Libro del alumno. Edelsa: Madrid, 2002.

. Cuaderno de ejercicios. Edelsa: Madrid, 2002.

SÁNCHEZ LOBATO, Jesús (org.). Español sin fronteras 1. SGEL: Madrid, 1997.

. Cuaderno de ejercicios. SGEL: Madrid, 1997.

. Español sin fronteras 2. SGEL: Madrid, 1998.

. Cuaderno de ejercicios. SGEL: Madrid, 1998.

. Español sin fronteras 3. SGEL: Madrid, 1999.

Cuaderno de ejercicios. SGEL: Madrid, 1999.

SANS BAULENAS, NEUS (org.). Gente 1. Curso comunicativo basado en el enfoque por tareas. Libro del alumno. Difusión: Barcelona, 1997.

Libro de trabajo y resumen gramatical. Difusión, Barcelona: 1997.

. Gente 2. Curso comunicativo basado en el enfoque por tareas. Libro del alumno. Difusión: Barcelona, 2000. 
REVISTA X, Curitiba, volume 14, n.2, p.145-157,2019.

. Libro de trabajo y resumen gramatical. Difusión, Barcelona: 2000.

. Gente 3. Curso comunicativo basado en el enfoque por tareas. Libro del alumno. Difusión: Barcelona 2001.

Libro de trabajo y resumen gramatical. Difusión, Barcelona: 2002.

Rápido, Rápido. Libro del alumno. Curso intensivo de español. Difusión: Barcelona, 2002.

SANZ, Bernardo (org.). Método Everest de español para extranjeros. Nivel Inicial. Libro del alumno. Universidad de Valladolid: Valladolid, 2001.

. Libro de ejercicios, Universidad de Valladolid: Valladolid, 2001.

. Método Everest de español para extranjeros. Nivel Intermedio. Libro del alumno. Universidad de Valladolid: Valladolid, 2001.

Libro de ejercicios, Universidad de Valladolid: Valladolid, 2001.

. Método Everest de español para extranjeros. Nivel Superior. Libro del alumno. Universidad de Valladolid: Valladolid, 2001.

Libro de ejercicios, Universidad de Valladolid: Valladolid, 2001. 